\title{
Zum Patientenbild in der Psychiatrie
}

\section{Klinischer Problemaufriss und historische Perspektive}

\author{
R. Borbé ${ }^{1}$, T. Müller ${ }^{2}$ \\ ${ }^{1}$ Allgemeine Psychiatrie und Psychotherapie Ravensburg, Klinik I für Psychiatrie und Psychotherapie der Universität \\ Ulm; ${ }^{2}$ Forschungsbereich Geschichte und Ethik in der Medizin, ZfP Südwürttemberg, Klinik I für Psychiatrie und Psy- \\ chotherapie der Universität Ulm
}

\section{Schlüsselwörter}

Patientenbild, Arztbild, Arzt-Patienten-Beziehung, Historiografie

\section{Zusammenfassung}

Die Beziehungsgestaltung zwischen Arzt und Patient wird von Bildern und Rollenzuschreibungen beeinflusst. Während es vielfältige Auslassungen zum Arztbild gibt, fehlt aber ein solches auf Patientenseite. Gerade in der Psychiatrie als "sprechendem Fach" aber auch wegen deren ordnungspolitischem Auftrag ist es für den Arzt wichtig, zu reflektieren, welches Bild er von seinem Gegenüber hat und dass dieses Bild verschiedenen Einflüssen unterworfen sein kann.

Die Literatursuche zum Patientenbild ergab, anders als zum Menschenbild, nur wenige Publikationen. In diesen wird ein "philanthropes" Patientenbild dem paternalistischen gegenübergestellt. Die Entscheidungsgewalt sei dem Patienten zugewiesen, der damit aber auch die Rolle eines selbstverantwortlich-mitwirkenden erhalte. Die geringe Publikationsdichte zum Patientenbild zeigt auch, dass die-

Korrespondenzadresse

Dr. med. Raoul Borbé, MHBA

Allg. Psychiatrie und Psychotherapie Ravensburg

Klinik I für Psychiatrie und Psychotherapie der Universität Ulm, Weingartshofer Str. 2, 88214 Ravensburg

Tel. 0751/76012216, Fax 0751/76012615

Raoul.Borbe@ZfP-Zentrum.de se Zuschreibungen in der Versorgungsrealität bisher kaum wahrgenommen wurden. Psychiatriehistorisch ist dies gut nachvollziehbar, da erst Mitte des 20. Jahrhunderts die Interessen und der Wille des Patienten sowohl berufsständische als auch politische Resonanz erfuhren. Klassische medizinhistorische Methoden blendeten dies weitgehend aus, während neuere Ansätze, wie die Patientengeschichte, die Auswertung von Selbstzeugnissen oder Pathografien die Wirklichkeit des Patienten besser abbilden.

\section{Keywords}

Picture of the patient, picture of the doctor, doctor-patient-relationship, history of psychiatry

\section{Summary}

The creation of the relationship between doctors / therapists and their patient is both influenced and limited by culturally bound images and attributed role patterns. While the

Picture of the patient in psychiatry

Nervenheilkunde 2018; 37: 723-728

eingegangen am: 14. Mai 2018

angenommen am: 30. Mai 2018 prospect of how "to be a good doctor" has been continuously developed and often reframed, a lack of prospect is obvious until today, of how therapists should perceive their patient. In the medical discipline of psychiatry this lack is especially obvious - not just for legal challenges, but mainly because of the high impact of communication, characteristic of this field of medicine, challenging us to reflect upon our prospect of the one seeking for help, diagnosis, and treatment. Researching publications on the matter reveals that research on communicational aspects of the doctor-patient-relationship hardly touches our prospect of the patient, thus sharply contrasting frequent debate on, for example, "the human being". The few contributions available focus on the comparison of a "philanthropic" notion of the prospect of the patient versus a paternalistic one. In these publications, decision-making is often attributed to the patient, however referring responsibility to his or her side as well.

In historical perspective, it becomes evident that both interest and will of patient have been put into the focus of political and professional attention no earlier than the mid$20^{\text {th }}$ century. Even the historiography of psychiatry neglected "the patient-view" for a long time, while more recent methodology profits from integrating the analysis of the patient's voice, related ego-documents and the reality of the ill.
In der täglichen Arbeit mit Patienten sind wir geleitet von einer Vielzahl externer und interner Einflüsse: Leitlinien, Leitbilder, Inhalte der Ausbildung, hierarchisch-institutionelle und eine Vielzahl anderer Faktoren bestimmen das Verhältnis ärztlich Tätiger zum Patienten. Das Arzt-Patientenverhältnis, die Beziehungsgestaltung zwischen Arzt und Patient, hat unzweifelhaft einen sehr hohen Stellenwert für jegliche Thera- pie und ihren Erfolg. Es ist vielfältig beschrieben worden (1). Die Bedeutung dieser Beziehung variiert naturgemäß in Abhängigkeit von der Diagnose und der Tatsache, ob es sich um eine akute oder eine chronische Erkrankung handelt. Sie variiert beispielsweise zwischen der Situation einer akuten, ohne zeitliche Verzögerung notwendigen Intervention bei einem komatösen Patienten einerseits oder der manchmal jahrelangen ärztlichen Begleitung eines Dialysepatienten andererseits. Dementsprechend würde man der Beziehungsgestaltung mit Blick auf Behandlungserfolg und Patientenzufriedenheit in diesen Fällen auch einen sehr differenten prädiktiven Wert beimessen. Anders bei psychischen Störungen: Hier ist - nicht allein weil es sich um ein sogenanntes „sprechendes Fach" handelt - die Beziehungsge- 
staltung bereits bei der ersten Begegnung von großer Wichtigkeit, auch weil ein nicht geringer Teil der Patienten und ihre Angehörigen erhebliche Vorurteile gegenüber der institutionalisierten Psychiatrie haben. Der Einfluss historischer Gegebenheiten ist bis heute offenkundig. Die Frage, was uns bei unserem täglichen Arbeiten mit Menschen mit psychischer Störung leitet, ist damit auch eine Kernfrage der Beziehungsgestaltung zwischen therapeutisch Tätigen und Patienten in der Psychiatrie.

Ausgangspunkt unserer Betrachtung ist die Tatsache, dass ein Verständnis der Arzt-Patient-Beziehung in der Medizin auf sehr differenzierte, zum Teil sehr traditionsreiche Konzepte eines „Arztbildes“ zurückgreifen kann (2), unsere Vorstellungen von einem - gar konsentierten - „Patientenbild" jedoch bis dato in nicht ausreichender Präzision reflektiert und formuliert wurden, die Definition eines Patientenbilds somit auch einer gewissen Beliebigkeit der jeweiligen therapeutischen Seite anheimgestellt war und ist.

Dieses Missverhältnis ist allein medizintheoretisch kaum tragbar. Denn das Patientenbild als Variante des populären Themas „Menschenbild“ (3) ist implizite Leitstruktur des Umgangs mit dem Patienten für die Behandelnden im medizinischen Alltag und daher von zentraler Bedeutung (4). Implizit fließt das Bild, das sich therapeutisch Tätige vom kranken Menschen machen, in Therapieleitlinien, gesetzliche Regelungen und viele weitere Dokumente von hoher medizinischer oder juristischer Bedeutung - aber auch in die Gestaltung von Versorgungsstrukturen - ein.

Im Folgenden soll daher verdeutlicht werden, auf welch vielfältige Art und Weise Vorstellungen vom kranken Menschen Eingang in zentrale medizinische, insbesondere psychiatrisch-psychotherapeutische, wie auch juristische Konstrukte, Handlungsanweisungen und nicht zuletzt: Gesetze, finden. Unsere Reflektionen sollen also nicht allein die klinische, oder alternativ die ökonomische Seite der Behandlung des Patienten beleuchten (5), vielmehr ist ein umfassendes und "unfragmentiertes" Patientenbild (6) als eine wesentliche Grundlage der Arzt-Patient-Beziehung zu betrachten. Eine Definition des Begriffs Patientenbild soll der Auffassung der Autoren zufolge also möglichst umfassend gedacht werden, ohne hierbei an Klarheit zu verlieren.

In die Analyse unserer Konzepte zum Aspekt des Patientenbilds fließen Selbstund Fremdbilder des Patienten ein (7). Das Patientenbild ist nicht minder auch eine Grundlage der Diskussion zur Selbstbestimmung des Patienten vor dem Hintergrund beispielsweise der UN-Behindertenrechtskonvention (8). Das Patientenbild beeinflusst unsere Vorstellungen, auch unsere theoretischen Grundlagen und praktischen Anwendungen von Patientenautonomie (4), auch diejenigen von Teilhabe der Patienten. Auch ist ein kommunizierbares und definiertes Patientenbild notwendige Voraussetzung für die Erstellung von Behandlungsvereinbarungen oder Patientenverfügungen $(9,10)$. Es ist nicht zuletzt Grundlage von Urteilen des Bundesverfassungsgerichts, oder anderer Stellungnahmen, wie z. B. zum Thema der Zwangsbehandlung - hier: in der Psychiatrie (11). Eingriffe in das Selbstbestimmungsrecht des Patienten sind Gegenstand aktueller und wohl auch zukünftiger Debatten und Aushandlungsprozesse, haben jedoch selbst bereits eine beindruckende und wechselhafte Geschichte (12).

Die Behandlung der Frage, weshalb Vorstellungen von Patienten bis heute weit weniger konzise und verlässliche Formen aufweisen, verweist auf die historische Perspektive: Die „klassischen“, psychiatriehistorisch relevanten Repräsentationen der psychiatrischen „Wirklichkeit" des Patienten, des Bildes vom Patienten selbst, werden in Erinnerung gerufen, die ein eher fremdbestimmtes Patientenbild in der Vergangenheit in Klinik wie medizinhistorischer Perspektive befördert haben (13-15). Die Erfahrung medizinischer Verbrechen kommt in der deutschen Geschichte (16), auch in der Medizingeschichte derjenigen Länder, die sich im Einflussbereich deutscher Besatzung während des Zweiten Weltkriegs befanden, in nachvollziehbarer Weise eine besondere und prägende Rolle zu („Nürnberger Kodex“). Rituale der Erinnerung an Patienten als Opfer interferieren ebenfalls mit gegenwärtigen Auffassungen zum Patientenbild (17).

Ausreichend Grund, sich näher und in kritischer Form mit den Bildern zu befas- sen, die wir uns von Patienten machen. Dies insbesondere, da die circa 200-jährige Geschichte der sich institutionalisierenden westeuropäischen Psychiatrie beziehungsweise deren Historiografie diesen Gegenstand erst seit den 1970er-Jahren als dem Kanon forschungswürdiger Gegenstände zugehörig betrachtet.

\section{Zum Begriff des Patienten- bildes}

Das Patientenbild beschreibt analog zum Menschenbild (3) das Bild, das wir uns vom Patienten machen, das veränderbar ist und vielfältigen Einflüssen unterliegt. Dieses Konzept integriert vielfältige Anteile, beispielsweise im Sinne eines in einer Fachgesellschaft konsentierten Patientenbilds. Es ist damit ein implizites und konstruiertes Bild, das unsere Haltung, unsere Herangehensweise, unsere Beziehungsaufnahme mit dem Patienten auf verschiedenen Ebenen wesentlich beeinflusst. Da dies auch mit den Aspekten von Haltung oder Einstellung interferiert, steuert es unser Handeln auch unbewusst mit. Dies unterstreicht die Notwendigkeit einer Auseinandersetzung mit Patientenbildern in der Psychiatrie und somit zunächst auch dem jeweils eigenen Patientenbild eines jeden therapeutisch Tätigen.

\section{Exemplarische Patienten- bilder in der Medizin}

Die Recherche nach Definitionen eines Patientenbilds in der Literatur gestaltete sich schwierig. Fachspezifische Suchmaschinen, wie PubMed verzeichnen für den deutschen Begriff keine Treffer, die Suche anhand einer englischen Übersetzung analog zum Menschenbild, also bspw. „concept of patient" oder abgewandelte Formen ergab ebenfalls keine Treffer. Es wurden im Rahmen der Recherche zum vorliegenden Beitrag daher auch allgemeine Suchmaschinen verwendet. Dabei fanden sich drei deutschsprachige Arbeiten, die mit dem Begriff des Patientenbildes arbeiten:

Die Sozialwissenschaftlerin Katrin Pöppel beleuchtet in ihrer Arbeit den „Wertewandel des sozialen Dienstleister Kranken- 
haus" (18) vor dem Hintergrund einer Analyse zum Patientenbild. Dabei setzt sie dem paternalistischen ein "philanthropes“ Patientenbild gegenüber, das sie aus den Persönlichkeits- und Selbstbestimmungsrechten westlicher Gesellschaften ableitet. Sie fand in ihrer Untersuchung eine entsprechende Werteorientierung in den Leitlinien der von ihr untersuchten Krankenhäuser, jedoch nicht deren Entsprechung in den ,untersuchten Maßnahmen organisationaler Zuwendung".

Der Philosoph Otfried Höffe spricht „(...) bewusst nicht vom ärztlichen, sondern vom medizinischen, Arzt und Patienten umgreifenden Menschenbild“ (19). Ausgehend von Aristoteles, der in seiner Metaphysik „(...) erklärt (...): Der Arzt heilt nicht einen Menschen, sondern einen Einzelnen“ sieht Höffe hier Bausteine „(...) sowohl für das Arzt- als auch das Patientenbild“: der Mensch sei erstens krankheitsanfällig, deshalb zweitens hilfsbedürftig und drittens sei ein anderer, der Arzt, hilfsfähig. Vor allem aber zählt viertens der Einzelne“. Höffe fügt diesen vier Bausteinen die Patientenhoheit hinzu. Zwei weitere Bausteine erweitern noch die Beschreibung des Mediziners: die Wissbegierigkeit und das Allmachtsverlangen, das „auf unschuldige Weise natürlich" sei. Es resultiert ein Bild, aufbauend auf Aristoteles, das die notwendigen Voraussetzungen für ein sich gegenseitig bedingendes Patienten-ArztVerhältnis skizziert. Der Begriff der Patientenhoheit weist dem Patienten in dieser Beziehung die Entscheidungsgewalt zu.

Die hinsichtlich der diesem Beitrag zugrunde liegenden Fragestellung differenzierteste Abhandlung liefert unseres Erachtens der schweizerische Autor Werner Widmer, der neben dem Selbst- auch ein Fremdbild von Patienten im Spital untersucht und im Weiteren mehrere prägnante Patientenbilder skizziert (7):

- der Hilfsbedürftige/Fürsorgeempfänger,

- der Fall,

- der Kunde,

- der selbstverantwortlich Mitwirkende.

All diese Patientenbilder treffen zweifelsfrei auch auf die Psychiatrie zu. Ergänzen müsste man noch, historisch an erster Stelle, den „Irren“, der weggesperrt werden muss (20).
Der ehemalige baden-württembergische Sozialminister Andreas Renner konstatierte 2005 in einem Grußwort zum 100-jährigen Jubiläum des Nordbadischen Zentrums für Psychiatrie in Wiesloch: „Die Psychiatrie ist heute geprägt von offenen Türen und einem humanen Patientenbild“, die frühere Verwahrpsychiatrie sei überwunden (21).

\section{Psychiatriehistorisch relevante Repräsenta- tionen der Wirklichkeit}

Die geringe Bedeutung, die dem Patientenbild augenscheinlich in aktuellen medizinischen Publikationen beigemessen wird, wirft die Frage auf, ob dies ein aktuelles Phänomen ist oder ob diese Perspektive auch medizinhistorisch nur wenig Beachtung fand. Der enge Begriff des Patientenbilds ist dafür zunächst nicht geeignet, da sich vielmehr die berechtigte Frage stellt inwieweit der Patient - und ohnehin der psychisch kranke Mensch - in früheren Zeiten überhaupt im Zentrum der Betrachtung stand?

Die Interessen und den Willen von Patienten unhinterfragt in den Mittelpunkt der ärztlichen Behandlung zu stellen, erhält jenseits paternalistischer Auffassungen seitens der heilenden Berufe erst in der zweiten Hälfte des 20. Jahrhunderts Eintritt in allgemeine Auffassungen des Berufsstands sowie gesetzliche Rahmenbedingungen zur ärztlichen Praxis. Kurioserweise werden auch in der Historiografie der Medizin der Patient selbst und seine individuelle Perspektive auf Krankheit und Leben erst sehr spät Gegenstand der Forschung. Die „Patientengeschichte" wurde demgemäß erst sehr spät kanonisiert als berechtigtes Forschungsfeld.

Die im Sinne der Genese des Fachs Medizingeschichte ursprünglichen, auch im deutschen Sprachraum klassisch-medizinhistorischen Methoden und Ansätze des Fachs, die dem Interesse der Patientengeschichte vorausgingen, können im Rahmen dieses Beitrags nur skizziert werden. Ihre Konzepte können hier nur kursorisch aufgeführt werden. Es sind dies im Wesentlichen: - die sogenannte Institutionsgeschichte,

- die positivistische ,Fortschritts-'Geschichte medizinischer Entdeckungen,
- die Auswertung ärztlicher Behandlungs- und Verlaufsberichte oder

- die ungezählten Beispiele biografischer, hagiografischer Geschichte des medizinischen Personals, in einer selbstreferenziellen, positivistischen Perspektive, die, trotz ihrer Vielfalt, in ihrer Gesamtheit oft mit dem angelsächsischen Begriff der "Whig History" bezeichnet werden (22).

Die genannten, in der frühen Medizinhistoriografie weit verbreiteten und repräsentativen Methoden trugen sämtlich dazu bei, dass Patienten entweder kein Gegenstand der Untersuchung waren, oder lediglich in Form statistischer Daten Erwähnung fanden. Oder - im Falle der Auswertung von Krankenakten: Die Vitae der Behandelten aus Sicht des therapeutischen Personals untersucht wurden. Dies zumindest in den vielen Fällen, in denen auf die Auswertung von Ego-Dokumenten verzichtet wurde.

Man darf weiterhin sagen, dass die handwerkliche Ausrichtung der (deutschsprachigen) Medizingeschichte entlang den genannten Traditionen der Geschichtsschreibung auch mit der Entstehung der Medizingeschichte aus der Klinik heraus zu erklären ist: Wesentliche Anregungen zur wissenschaftlichen Beschäftigung mit der Geschichte der eigenen Tätigkeit kamen aus der Klinik selbst, die ersten Akteure waren häufig ehemals klinisch Tätige. Eine deutliche Erweiterung des methodologischen Spektrums erfolgte erst im letzten Viertel des 20. Jahrhunderts. Dies wesentlich durch in der Allgemeingeschichte entwickelte Ansätze und Konzepte der Forschung. Eine geringe Beachtung der Patientenperspektive war demgemäß auch in der Medizinhistoriografie, und dies bis in die 1960er-Jahre hinein, die Regel. Interessant ist darüber hinaus hier die Beobachtung, dass auch solche Methoden und Ansätze, die sich ganz allgemein und zu Recht als innovativ für den relevanten Wissenschaftsbereich erwiesen, für die im Zentrum stehenden Fragen zum Patient, seiner Auffassung, seines Willens, auch zu seiner Geschichtsschreibung hinter diesem Anspruch dennoch weiterhin zurück blieben. Ein Beispiel stellt die durch Michel Foucault inspirierte Psychiatriegeschichte dar. 
Es ist einerseits Michel Foucault und dem immensen Einfluss seines Wirkens zu verdanken - mit Veröffentlichung seiner 1961 verteidigten Qualifikationsarbeit, die in deutscher Sprache erstmals 1969, und überarbeitet 1995 erschien (Folie et déraison: Historie de la folie à l'âge classique) dass der Geschichte der Psychiatrie, dieser scheinbar so anrüchigen und scheinbar immer am Rande der Legalität agierenden medizinischen Subdisziplin und ihren Akteuren ab diesen Jahren eine zuvor nie dagewesene Bedeutung und Attraktivität zukam (23-25). Das Aufbegehrende in Foucaults Werk folgte nachvollziehbaren emotionalen Impulsen, angestrebtem Wissenszugewinn und intellektueller Entwicklung gleichermaßen. Derart zum seriösen Gegenstand der Geschichtsforschung geworden, kam der Psychiatrie und ihrer Geschichte durch die zum Teil mutigen, zum Teil einfach auch gewagten und jedenfalls medizinkritischen Thesen Foucaults mehr Aufmerksamkeit zu. Die Kritik an Foucaults Position seitens der Geschichtswissenschaften wiederum ist ausreichend beschrieben worden (23-29) und nicht erneut wiederzugeben. Foucault selbst revidierte später durchaus zentrale Aspekte seiner frühen Konzeptionen. Doch auch Foucaults Arbeiten entbehrten annähernd völlig der Perspektive von Patienten. Es mag mit der Grenzenlosigkeit der Wirkmacht zu tun haben, die Foucault „totalen Einrichtungen" wie der Anstalt zuschrieb, dass ihre Insassen (und Opfer) - deren Situation uns hier interessiert - nicht als Handelnde auftraten (30). Ego-Dokumente wurden auch in dieser wirkmächtigen Psychiatriegeschichtsschreibung weiterhin systematisch ausgesondert, da sie keine veritable Quelle darstellten (31).

\section{Jüngere Ansätze und Methoden der Forschung und des (Be-)Schreibens}

Die akademische Medizinhistoriografie emanzipierte sich erst in den 1970er-Jahren von der alleinigen Darstellung aus ärztlicher Perspektive. Sie integrierte Methoden und Ansätze zur Erforschung der Medizingeschichte, die den Kranken selbst, sein Leben, seine Zeugnisse ins Spiel brachten.
Dies gilt insbesondere auch für die Geschichte der Psychiatrie. Nach ihrer „Entdeckung" und Integration in den Kanon historisch forschungswürdiger Gegenstände in den 1980er-Jahren wurde die Psychiatriegeschichte in den beiden folgenden Dekaden fast als eine „Schaubühne benutzt, um die (historiografischen) Konzepte und Praktiken von heute entweder $\mathrm{zu}$ kritisieren oder zu legitimieren“ (32). $\mathrm{Zu}$ den jüngeren Ansätzen und Methoden sind im Sinne der Fragestellung zu zählen:

- die Patientengeschichte,

- die Auswertung von Bildquellen, hier die Auswertung fotografischer Quellen,

- die Auswertung von Selbstzeugnissen, Ego-Dokumenten

- und deren für die Psychiatriegeschichte ausnehmend bedeutsamer Fall der Untersuchung künstlerischer Produktionen,

- pathografische und autopathografische Berichte als Quellen.

Zunächst die Patientengeschichte: Sie wurde vor allem durch zwei frühe Arbeiten inspiriert, von Buckley and Sander aus den USA 1974 einerseits sowie die deutlich bekanntere, englische Arbeit von Roy Porter 1985, die den einprägsamen und programmatischen Titel Doing History from Below hatte. Existierten Selbstzeugnisse von als psychisch krank zu bezeichnenden Menschen seit Jahrhunderten, und waren beispielsweise bereits auch aus der Antike überliefert, so erhielten diese trotz ihrer vormaligen Zugänglichkeit erst jetzt ihren Platz in der Historiografie (33). Analysiert und interpretiert wurden nun autobiografische (34) und autopathografische Berichte von Patienten oder ihre Briefe an Dritte (35). Auch öffentliche Pamphlete und Protestbrief-Sammlungen (36), von Patienten hergestellte Kunstwerke, sogar Kleidungsstücke und Alltagsgegenstände der Patienten, wie sie die berühmte PrinzhornSammlung in Heidelberg beinhalten (37), wurden interpretiert und anderes, in Krankenakten beinhaltetes Material wurde ebenfalls herangezogen (38). Natürlich waren geschlechtsspezifische Untersuchungen zu finden, wie Nolte 2003 gezeigt hat (39). Die Direktheit und Unvermitteltheit des seitens der Patienten bunt und vielfältig Produzierten, schien hier ein Garant für die Authentizität des historiografisch Reproduzierten zu sein. Die Wirklichkeit des Patienten wiederum schien hierdurch quasi greifbar (40). „Mit dem cultural turn der 1990er-Jahre verschob sich der Fokus. Nicht mehr die vermeintlichen Realitäten“, so formulierten die Organisatoren einer Tagung am Göttinger Lichtenberg-Kolleg, „sondern die kulturellen und symbolischen Interpretationskonstrukte standen (nun) im Zentrum des Interesses“. Wirklichkeit schien nicht mehr tatsächlich vorhanden, sondern war nun ein „kulturell geformtes und symbolisch repräsentiertes menschliches Konstrukt" (41).

Die Konzepte und Praktiken der Forscher interessieren sich dabei - in einem politisch und emotional stark aufgeladenen Diskurs - manchmal nur oberflächlich und diskontinuierlich für die Wirklichkeit des Kranken (13). Historische Quellen wurden zum Teil jeweils zwar „neu entdeckt“, ihre Nutzung und Auswahl läuft jedoch jederzeit Gefahr, den Anwendungsregeln und Implikationen eines weiteren, neuen wissenschaftlichen „turns“ nachgestellt zu werden. Jeweils neue Erzählweisen der Wirklichkeit des Kranken werden derart zyklisch generiert. Diese dominieren dann eher hegemonial die Anwendungspraxis, als dass methodologische Ko-Existenzen geschaffen würden, wie Ernst und Mueller dies in 2010 diskutiert haben (42). Ein Ausweg besteht hier unseres Erachtens in der Anpassung der gewählten Methoden an die vorhandenen Quellen, nicht einer umgekehrten Vorgehensweise.

In aktuellen medizinischen Debatten zum Thema des Patientenbilds, die bezüglich medizinethischer Aspekte häufig gut informiert erscheinen, müssen historiografische Debatten, Befunde und Erkenntnisse der Patientengeschichte hingegen vermehrt Eintritt finden. Die Perspektive von Patienten zu vernachlässigen, stellt ein Versäumnis dar: in der Geschichtsschreibung wie in der gegenwärtigen Praxis der Medizin.

\section{Diskussion}

Zeitgemäße, klinisch-psychiatrische Therapie versteht Behandlung zwischen $\mathrm{Pa}$ tienten und den sie Therapierenden demnach als partnerschaftliche, verbale oder 
non-verbale vertragliche Regelung. Wie diese kulturell diversifizierten vertraglichen Bindungen, diesseits wie jenseits juristischer Verbindlichkeiten, setzen auch andere weitere Prozesse im Umgang mit kranken Menschen voraus, dass unser Bild vom kranken und gesunden Menschen klar und reflektiert zur Anwendung kommt, d. h. in kommunikative Prozesse jedweder Art - als Teil dieser therapeutischen Beziehung - integriert wird.

Im Hinblick auf die aktuelle medizinische Praxis fällt jedoch auf, dass unser Bild vom Patienten weit weniger definiert und reflektiert zu sein scheint, als beispielsweise unser „Arztbild“, hinsichtlich dessen Definition eine ganze Reihe nationaler, kulturell gebundener oder international verbindlicher Formen und Normen bereits heute existieren. Dieser Beitrag plädiert für einen bewussten Umgang mit diesem Mangel des Status quo und versteht sich als Anstoß für eine Debatte, deren Ziel klarere Vorstellungen und damit Formulierungen zum Patientenbild sein sollen.

Das von Widmer skizzierte Patientenbild des „selbstverantwortlich-mitwirkenden" Patienten (7) erscheint breit anwendbar, da es dem zunehmendem Primat des Selbstbestimmungsrechtes in der Medizin nahe kommt. Es eignet sich daher zur Beschreibung eines Patientenbildes in der Psychiatrie. In den spezifischen Situationen einer akuten Eigen- oder Fremdgefährdung erfährt diese Generalisierung Einschränkung. Das Prinzip wird hierdurch jedoch nicht weniger gültig, sondern wird gewissermaßen temporär ausgesetzt. Entsprechende Sondergesetze, die die Zwangsbehandlung regeln, haben - im Tenor der Rechtsprechung des Bundesverfassungsgerichtes aus dem Jahr 2011 - gerade auch deshalb die Wiederherstellung des freien Willens, d. h. der Selbstbestimmungsfähigkeit als Ziel $(43,44)$. Die Akzeptanz dieser Selbstbestimmtheit, dieser „innere Wandel der Arzt-Patienten-Beziehung " wird in der Psychiatrie durchaus rezipiert (1), jedoch nicht im Sinne eines Patientenbildes beschrieben.

\section{Interessenkonflikt}

Es bestehen keine Interessenkonflikte.

\section{Literatur}

1. Helmchen H. Das Arzt-Patient-Verhältnis zwischen Individualisierung und Standardisierung. In: Berlin-Brandenburgische Akademie der Wissenschaften (Hrsg.). Berichte und Abhandlungen Band 11. Berlin 2006, 99-117.

2. Hess R. Das Arztbild im 21. Jahrhundert. In Kölner Schriften zum Medizinrecht 2009; 1: 117-120.

3. Fahrenberg J. Annahmen über den Menschen. Menschenbilder aus psychologischer, biologischer, religiöser und interkultureller Sicht. Heidelberg: Asanger 2004.

4. Borbé R. Patientenautonomie in der Psychiatrie. In Wiesemann C, Simon A (Hrsg.). Patientenautonomie. Theoretische Grundlagen, Praktische Anwendungen. Münster: Mentis 2013, 190-203.

5. Schernus R. Der Geist der Psychiatriereform und das Diktat der Ökonomie. Kerbe 2008; 2: 11-15.

6. Böker W. Der fragmentierte Patient. Deutsches Ärzteblatt 2003; 1: 21-24.

7. Widmer W. Selbst- und Fremdbild der Patientinnen und Patienten im Spital. Schweizerische Ärzte-Zeitung 2005; 49: 2706-2710.

8. UN-Behindertenrechtskonvention (UN-Behindertenrechtskonvention, amtliche deutsche Übersetzung). www.un.org/Deptsgerman/ueberein kommen/ar61106-dbgbl.pdf.

9. Borbé R, Jaeger S, Borbé S, Steinert T. Anwendung psychiatrischer Behandlungsvereinbarungen in Deutschland. Nervenarzt 2012; 83: 638-643.

10. Vollmann J. Patientenverfügungen von Menschen mit psychischen Störungen. Gültigkeit, Reichweite, Wirksamkeitsvoraussetzung und klinische Umsetzung. Nervenarzt 2012; 83: 25-30.

11. Steinert T. DGPPN-Leitlinie; Steinert, Tilman: Anti-Folter-Komitee und Architektur psychiatrischer Kliniken. Psychiatrische Praxis 2012; 39: 243-247.

12. Noack T. Eingriffe in das Selbstbestimmungsrecht des Patienten. Juristische Entscheidungen, Politik und ärztliche Positionen, 1890-1960. Frankfurt: Mabuse 2004.

13. Müller T. Die Wirklichkeit des Kranken. Medizinhistorische Wirklichkeitsrepräsentationen am Beispiel der Psychiatrie und ihrer Historiker /-innen. In: Haas, Stefan und Wischermann, Clemens (Hrsg.). Die Wirklichkeit der Geschichte. München: Fink 2015; 127-148.

14. Müller T. Zentrum und Peripherie aus der Perspektive medizinhistorischer Forschung. Das Beispiel der Medizin im Nationalsozialismus. In: Müller T (Hrsg.). Zentrum und Peripherie in der Geschichte der Psychiatrie. Regionale, nationale und internationale Perspektiven (= Reihe Kulturanamnesen). Stuttgart: Franz Steiner 2017; 221-237.

15. Brückner B, Röske T, Rotzoll M, Müller T. Geschichte der Psychiatrie „von unten“ - Entwicklung und Stand der deutschsprachigen Forschung, 2006-2016 (in Vorbereitung).

16. Müller T, Schmidt-Michel PO. Die Begegnung unserer Klinik in Weissenau mit der NS-Vergangenheit und das Denkmal der grauen Busse. In Müller $\mathrm{T}$ et al. (Hrsg.). Psychiatrie in Oberschwaben. Die Weissenau zwischen Versorgungs- funktion und universitärer Forschung. Zwiefalten: Verlag Psychiatrie 2017; 371-375.

17. Müller T et al. (Hrsg.): Vergangen? Spurensuche und Erinnerungsarbeit - Das Denkmal der grauen Busse. Zwiefalten: Verlag Psychiatrie 2017.

18. Pöppel K. Wertewandel beim sozialen Dienstleister Krankenhaus. Eine Analyse zum Patientenbild. Frankfurter Schriften zur Gesundheitspolitik und zum Gesundheitsrecht. Frankfurt: Peter Lang 2008 .

19. Höffe O. Patientenwohl im Zeitalter der Allmacht. In: Blum HE, Haas R. (Hrsg) Publikationen der Jung-Stiftung für Wissenschaft und Forschung Über das Menschenbild in der Medizin. Stuttgart: Thieme 2004; 26-33.

20. Schott H, Tölle R. Geschichte der Psychiatrie. Krankheitslehren - Irrwege - Behandlungsformen. München: C.H. Beck 2006.

21. Psychiatrisches Zentrum Nordbaden (PZN). Report 2004/2005 - Jubiläumsausgabe. Online: https://www.pzn-wiesloch.de/fileadmin/userupload/Wiesloch/Report_PDFe_nur_UK/ PZNGB2004.pdf,

22. Butterfield $H$. The Whig Interpretation of History. New York City: Bell \& Sons 1931.

23. Scull AT. Museums of Madness. New York City: Viking 1979.

24. Scull AT (ed.). Madhouses, Mad-Doctors and Madmen. The Social History of Psychiatry in the Victorian Era. Philadelphia: University of Philadelphia Press 1981.

25. Wear A. Medicine in society. Cambridge: University Press 1992; 9-10.

26. Porter R. The Patient's View. Doing Medical History from Below. Theory and Society 1985; 14(2): 175-198.

27. Porter R. A Social History of Madness: Stories of the Insane. London: Weidenfeld \& Nicholson 1987.

28. Digby A. Madness, Morality and Medicine. A Study of the York Retreat, 1796-1914. New York: Cambridge University Press 1985.

29. Koopman C. Foucault across the disciplines: introductory notes on contingency in critical inquiry. History of the Human Sciences 2011; 24: 1-12.

30. Osten P (Hrsg.). Patientendokumente. Krankheit in Selbstzeugnissen (=Medizin, Gesellschaft und Geschichte, Beiheft 35). Stuttgart: Franz Steiner 2010; 7.

31. Wehler HU. Die Herausforderung der Kulturgeschichte. München: Beck 1998; 45-95.

32. Engstrom E. Heterotopischer Wahnsinn: Aktuelle Forschungsansätze in der Psychiatriegeschichte. Jahrbuch für Universitätsgeschichte 2009; 12: 271-275.

33. Melling J, Forsythe B. The Politics of Madness. The state, insanity and society in England, 1845-1914. Routledge Studies in the social history of medicine. Abingdon and New York 2006; 20: 176-203.

34. Spuhler G. Gerettet - zerbrochen. Das Leben des jüdischen Flüchtlings Rolf Merzbacher zwischen Verfolgung, Psychiatrie und Wiedergutmachung. Zürich: Chronos 2011.

35. Dinges M, Barras V (Hrsg.). Krankheit in Briefen im deutschen und französischen Sprachraum, 17.-21. Jahrhundert. Stuttgart: Franz Steiner 2007.

36. Brink C. Grenzen der Anstalt. Psychiatrie und Gesellschaft in Deutschland 1860-1980. Göttingen: Wallstein 2010. 
37. Ankele M. Alltag und Aneignung in Psychiatrien um 1900. Selbstzeugnisse von Frauen aus der Sammlung Prinzhorn. Weimar: Böhlau 2009.

38. Ledebur S. Schreiben und beschreiben. Zur epistemischen Funktion von psychiatrischen Krankenakten, ihrer Archivierung und deren Übersetzung in Fallgeschichten. Berichte zur Wissenschaftsgeschichte 2011; 34: 102-124.

39. Nolte K. Gelebte Hysterie. Erfahrung, Eigensinn und psychiatrische Diskurse im Anstaltsalltag um 1900. Frankfurt: Campus 2003.
40. Wolff E. Patientenbilder: zur neueren kulturwissenschaftlichen Gesundheitsforschung. In: Unterkircher A, Bodner R, Sohm K (Hrsg.). Medikale Kulturen. Innsbruck: University Press 2008, 24-38.

41. Haas S, Wischermann C (Hrsg.). Die Wirklichkeit der Geschichte. Stuttgart: Franz Steiner 2015.

42. Ernst W, Mueller T (eds.). Transnational Psychiatries. Social and cultural histories of psychiatry in comparative perspective, c. 1800-2000 Newcastle on Tyne 2010, $2^{\text {nd }}$ edition 2015.
43. Bundesverfassungsgericht: http://www.bundes verfassungsgericht.de/SharedDocs/Downloads/ DE/2011/03/rs20110323_2bvr088209.pdf?_blob= publicationFile\&v $=1$

44. Maio G. Mittelpunkt Mensch: Ethik in der Medizin. Ein Lehrbuch. Stuttgart: Schattauer 2012. 


\section{Zertifizierte Fortbildung}

Fragen zum Thema „Zum Patientenbild in der Psychiatrie”

1. Welche Aussage zum Patientenbild ist nicht richtig?

a) Das Patientenbild beeinflusst die Beziehungsgestaltung zum Patienten.

b) In der Literatur wird meist ein „philanthropes" Patientenbild einem paternalistischem gegenübergestellt.

c) Ökonomische Interessen von Klinikkonzernen sind frei von Patientenbildern.

d) Patientenbilder entsprechen häufig dem vorherrschenden Menschenbild.

e) Historische Einflüsse bestimmen das Patientenbild mit.

2. Definitionen des Patientenbildes sind im Internet und der Sekundärliteratur nicht ausreichend belegt, weil ...

a) zu wenig über psychisch Kranke berichtet wird.

b) nur wenige Bundesbürger Erfahrung mit psychischer Erkrankung machen.

c) das Thematisieren von psychischer Erkrankung und ihren Folgen nicht unabdingbar zu einer verbindlichen Definition des Patientenbildes führt.

d) zu wenige Experten sich zu dieser Frage äuBern wollen.

e) die Stigmatisierung psychischer Erkrankung bis heute eine Definition des Patientenbilds verhindert.

3. Welche der Aussagen ist nicht zutreffend? Das Bild, das Therapierende sich von Patienten machen, ist historisch zu kontextualisieren, weil ...

a) viele Erkenntnisse zu psychischen Erkrankungen sich über eine zeitliche Dauer als unveränderlich erweisen.

b) die Wahrnehmung psychischer Erkrankung sich über die Zeit verändern kann.

c) historische und kulturelle Gegebenheiten (z. B. Kriege) die Wahrnehmung von psychischen Erkrankungen verändern können.

d) verschiedene Kontexte auch gesundheitspolitisch divergierende Perspektiven auf den Patienten aufweisen können.

e) wissenschaftliche Kontextualisierung und eine Transparenz der Definition des Patientenbilds auch internationale Vergleichbarkeit ermöglichen können.
4. Welche der Aussage ist nicht zutreffend: Politische Informierung erhält die Debatte um ein Patientenbild im Gesundheitswesen durch folgende Seiten:

a. die UN-Behindertenrechtskonvention.

b. den Hippokratischen Eid.

c. medizinethische Beiträge.

d. Patientenvertreter und -fürsprecher.

e. das Bundesteilhabegesetz.

5. Welche der Aussagen ist richtig? Seit wann gehört die Psychiatriegeschichte zu den historisch forschungswürdigen Gegenständen?

a. Seit den 1920er-Jahren.

b. Seit der Antike.

c. Die Psychiatriegeschichte ist nicht forschungswürdig.

d. Seit den 1980er-Jahren.

e. Seit den 1960er-Jahren.

6. Zu den klassisch-medizinhistorischen Ansätzen, die dem Interesse der Patientengeschichte vorausgingen zählt nicht:

a) Biografien des medizinischen Personals.

b) Auswertung ärztlicher Behandlungsberichte.

c) mikrohistorische Untersuchungen.

d) die sog. Institutionsgeschichte.

e) die positivistische Fortschrittsgeschichte medizinischer Entdeckungen.

7. Auf welche Patientenbilder wird in dem vorliegenden Beitrag nicht Bezug genommen?

a) der Fürsorgeempfänger.

b) der Kunde.

c) der Fall.

d) der Partner.

e) der selbstverantwortlich Mitwirkende.

8. Die Arzt-Patienten-Beziehung ist in heutiger Zeit gekennzeichnet durch

a) die Betonung des ärztlichen Paternalismus.

b) einen selbstverantwortlich-mitwirkenden Patienten.

c) ein fragmentiertes, differenziertes Patientenbild.
CME-Fortbildung online

Die Teilnahme an dieser durch die Ärztekammer Nordrhein zertifizierten CME-Fortbildung ist für 12 Monate ausschließlich online möglich. Zur Anmeldung gehen Sie bitte auf http://cme.thieme.de. Dort erfahren Sie auch den genauen Teilnahmeschluss. Es ist immer nur eine Antwort pro Frage zutreffend. Als Leser der Nervenheilkunde nehmen Sie kostenlos am CME-Programm teil. Je nach CME-Fortbildung erhalten Sie bis zu vier Fortbildungspunkte. Weitere Informationen zur Anmeldung und Registrierung finden Sie unter http://cme.thie me.de. Sie erhalten bei inhaltlichen und technischen Fragen tutoriellen Support.

VNR 2760512018154652820

d) ein Primat der Patientenautonomie auch bei akuter Eigen- oder Fremdgefährdung.

e) ein gleichberechtigtes, das Arztbild ergänzende Patientenbild.

9. Zu den jüngeren Ansätzen und Methoden historiografischer Konzepte gehören nicht

a) die Auswertung von Selbstzeugnissen.

b) die Biografien behandelnder Ärzte.

c) die Auswertung fotografischer Quellen.

d) die Patientengeschichte.

e) pathografische Berichte als Quellen.

10.Welche Eigenschaften, die sowohl ein Arzt- als auch ein Patientenbild maßgeblich beeinflussen, schreibt der Philosoph Otfried Höffe dem Arzt, bzw. Patienten nicht zu?

a) Hilfsbedürftigkeit

b) Hilfsfähigkeit

c) Unterwürfigkeit

d) Anfälligkeit für Krankheiten

e) Wissbegierigkeit 